TITLE:

\title{
Understanding of the intrinsic difference between normal- and perfluoro-alkyl compounds toward total understanding of material properties
}

\section{$\operatorname{AUTHOR}(\mathrm{S}):$}

Hasegawa, Takeshi

\section{CITATION:}

Hasegawa, Takeshi. Understanding of the intrinsic difference between normal- and perfluoro-alkyl compounds toward total understanding of material properties. Chemical Physics Letters 2015, 627:64-66

ISSUE DATE:

2015-05-01

URL:

http://hdl.handle.net/2433/218620

\section{RIGHT:}

(c) 2015. This manuscript version is made available under the CC-BY-NC-ND 4.0 license

http://creativecommons.org/licenses/by-nc-nd/4.0/; The full-text file will be made open to the public on 1 May 2017 in accordance with publisher's 'Terms and Conditions for Self-Archiving'.; この論文は出版社版でありません。引用の際に は出版社版をご確認ご利用ください。; This is not the published version. Please cite only the published version. 


\title{
Understanding of the intrinsic difference between
}

\section{normal- and perfluoro-alkyl compounds toward total understanding of material properties}

\author{
Takeshi Hasegawa ${ }^{\dagger 1}$ \\ ${ }^{1}$ Laboratory of Solution and Interface Chemistry, Division of Environmental Chemistry, Institute for \\ Chemical Research, Kyoto University, Gokasho, Uji, Kyoto 611-0011, Japan
}

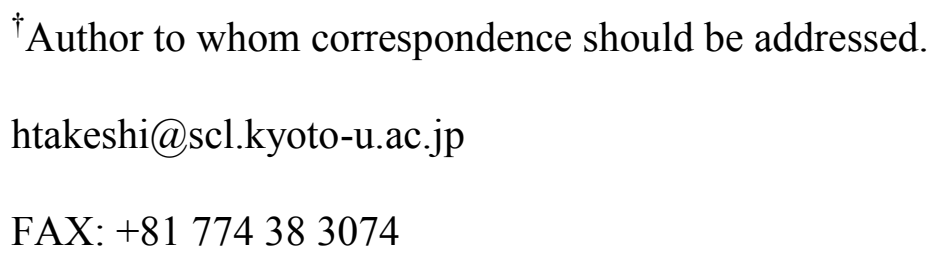




\begin{abstract}
Molecular interaction of perfluoroalkyl (Rf) compounds has long been discussed on an extended theory of normal alkyl compounds. When Rf-specific bulk properties depending on the molecular packing structure are taken into account, however, the conventional polarizability theory has a big inconsistency especially with the high melting point. Here, we present a new viewpoint to totally uniform the conventional theories for systematically accounting for the bulk properties of Rf compounds. With the organized theoretical framework, the conventional understanding based on polarizability proves to be partly true, but it misses the molecular orientation effect, which is specifically necessary for the Rf compounds.
\end{abstract}


Introduction: Understanding of unique bulk properties specific to perfluoroalkyl (Rf) compounds, ${ }^{1}$ which are represented by water- and oil-repellency, low dielectric permittivity, low chemical reactivity and high melting temperature, have long been an old and new unresolved issue in fundamental chemistry. Above all, the high melting temperature $\left(327^{\circ} \mathrm{C} \text { for PTFE }\right)^{2}$ is the most fundamental chemical property, straightforwardly implying that the Rf groups have intrinsically strong van der Waals forces between the groups. Although this strong interaction is widely recognized as 'fluorophilic effect, ${ }^{3}$ there has been very few physicochemical models accounting for the extraordinarily strong interaction. The stratified dipole-arrays (SDA) model ${ }^{4}$ recently proposed is the first chemical model to uniformly account for the bulk properties involving the fluorophilic effect, which has three outstanding characteristics that 1) dipoles play a dominant role instead of polarizability, 2) a single molecule and molecular aggregates are discussed in a distinguished manner, and 3) the mutual molecular orientation in an aggregate plays an inevitable role. To fully understand the entire theory, a bridging theory to the conventional approaches based on polarizability is necessary.

In the present paper, the perspective of the molecular interaction of both normal alkyl and $\mathrm{Rf}$ groups is provided based on the London's theory and electrodynamics, so that the reason why the conventional polarizability theory works well even for the $\mathrm{Rf}$ groups, although the dipole-dipole interaction plays the major role on the Rf compounds.

Discussion: Thus far, the chemical properties of an Rf group have mostly been discussed in an extended manner of a normal alkyl group: the molecular interacting property of normal alkyl groups 
relies on the London dispersion force, which seems reasonable. London theoretically deduced an apparent equation on quantum mechanics and electrodynamics that the van der Waals force comprises three distinct origins: the orientation effect, the induction effect and his dispersion effect. ${ }^{5}$ The "orientation effect" corresponds to the "dipole-dipole interaction" as a function of mutual orientation of the dipoles, which agrees with Keesom's theory deduced on classical mechanics. ${ }^{6}$ If the dispersion effect plays a dominant role, this dipole interaction effect can be ignorable in the van der Waals force for the normal alkyl groups, since the rest induction effect is known to be very weak for most cases. ${ }^{5}$ This expectation is true of a normal alkyl group, which can simply be examined as follows.

The three effects for a rigid dumb-bell shaped dipole have apparent analytical expressions as: ${ }^{5}$

$$
\begin{aligned}
& \bar{U}_{\text {orientation }}=-\frac{1}{3 R^{6}} \frac{\mu^{4}}{k_{\mathrm{B}} T}\left(1+3 \cos ^{2} \theta\right) \\
& \bar{U}_{\text {induction }}=-\frac{2}{R^{6}} \mu^{2} \alpha \\
& \bar{U}_{\text {dispersion }}=-\frac{3}{4 R^{6}} h v_{0} \alpha^{2}
\end{aligned}
$$

Here, the negative sign indicates that all the three effects work as attractive forces. The dipole moment and the molecular polarizability are denoted as $\mu$ and $\alpha$, respectively. The mutual orientation of two dipoles is represented by $\theta$. The inter-object length, $R$, can be removed out when the three effects are compared as a ratio, since $R^{-6}$ is commonly involved in the three equations. $h v_{0}$ is the zero-point energy of the electron vibration about the object, which can be replaced by the ionization energy within a good approximation. ${ }^{5}$ When the permanent dipole moment, $\mu=\mu_{0}$, has a random orientation, $\cos ^{2} \theta$ in Eq. (1) can be orientation-averaged ${ }^{5}$ to be $1 / 3$ to have: 


$$
\bar{U}_{\text {orientation }}^{\text {random }}=-\frac{2}{3 R^{6}} \frac{\mu^{4}}{k_{\mathrm{B}} T} .
$$

With the use of the bond parameters, ${ }^{7-9}$ the three effects between two $\mathrm{C}-\mathrm{H}$ groups are calculated by Eqs. (2) (4) as presented in Table 1. As expected, the van der Waals force among the $\mathrm{C}-\mathrm{H}$ groups is governed by the dispersion effect with a significant ratio of $89.6 \%$. In other words, a normal alkyl group is not influenced apparently by the weak dipole orientation effect (6.96\%).

On the other hand, the $\mathrm{C}-\mathrm{F}$ bond has a totally different character: the dipole-dipole interaction (orientation effect) works greatly, as the bond has a large dipole moment of $1.39 \mathrm{D},{ }^{7,10}$ which has 3.47 times larger than that of the $\mathrm{C}-\mathrm{H}$ bond $(0.40 \mathrm{D})$, and the orientation effect is a function of $\mu^{4}$. As a result, the dipole-dipole interaction between the $\mathrm{C}-\mathrm{F}$ bonds plays a dominant role in the van der Waals force with a significant ratio of $91.8 \%$; whereas the dispersion effect goes down to $5.03 \%$. The orientation effect of $61.5 \times 10^{-79} \mathrm{~J} \mathrm{~m}^{3}$ seems fairly large, but it is comparable to that of ammonia $\left(84 \times 10^{-79} \mathrm{~J} \mathrm{~m}^{3}\right),{ }^{5}$ which falls in the normal range of orientation effect.

Although the polarizability of the $\mathrm{C}-\mathrm{F}$ bond $\left(0.555 \times 10^{-30} \mathrm{~m}^{3}\right)$ is a little smaller than the $\mathrm{C}-\mathrm{H}$ one $\left(0.652 \times 10^{-30} \mathrm{~m}^{3}\right)$, as is often pointed out for explaining a lower refractive index, ${ }^{11}$ the decrease of the dispersion effect $\left(5.43 \times 10^{-79} \rightarrow 3.37 \times 10^{-79} \mathrm{~J} \mathrm{~m}^{3}\right)$ is thus ignorable in the van der Waals force between the $\mathrm{C}-\mathrm{F}$ groups. In this manner, the molecular interaction of $\mathrm{Rf}$ compounds is concluded to be governed by dipole moments, not by polarizability.

The intrinsic difference between the normal alkyl and Rf groups is not represented only by the interaction forces, but a more chemical characteristic. Since the mutual orientation of two $\mathrm{C}-\mathrm{X}$ groups, $\theta$, is involved in only Eq. (1), the conclusion above straightforwardly indicates that the 
"mutual dipole orientation of Rf groups" should also be a crucial factor to discuss the molecular aggregation property of a Rf compound; whereas the orientation can be put aside for a normal alkyl group. The SDA model ${ }^{4}$ points out that the helical Rf groups are spontaneously aggregated in a two-dimensional manner via the dipole-dipole interactions where the dipoles representing the $\mathrm{CF}_{2}$ groups are aligned linearly in a head-to-tail manner to make dipole arrays. The dipole-dipole orientation results in $\theta=0$ to make $\bar{U}_{\text {orientation }}$ be:

$$
\bar{U}_{\text {orientation }}^{\theta=0}=-\frac{4}{3 R^{6}} \frac{\mu^{4}}{k_{\mathrm{B}} T},
$$

which is twice larger than $\bar{U}_{\text {orientation }}^{\text {random }}$. This energetic stabilization thanks to the "linear dipole-dipole orientation" due to the SDA packing, the high melting point and the fluorophilic effect are readily understood (Figure 1).

Then, why has the polarizability been employed ${ }^{11}$ for elucidating the bulk properties thus far instead of using dipoles? For example, an Rf-specific character of "low electric permittivity $(\varepsilon)$," has long been attributed to the small polarizability of the $\mathrm{C}-\mathrm{F}$ bond, ignoring the large dipole moment. Since the conventional polarizability theory has been a hypothesis using no apparent physical equations, here we are organizing the theory as follows.

The polarizability, $\alpha$, is interrelated with the induced (not permanent) dipole moment, $p$, generated by an external electric field, $E$ :

$$
p=\alpha E
$$

Since $p$ is located on a single molecule, the polarizability is often called molecular (not bulk) polarizability. ${ }^{8,12}$ In a bulk matter, on the other hand, the collection of the induced dipole moments 
yields the polarization density, $P,{ }^{12}$ which is related to the electric field via the relative electric susceptibility, $\chi_{\mathrm{r}}$ :

$$
P=\varepsilon_{0} \chi_{\mathrm{r}} E
$$

Since Eqs. (5) and (6) look similar to each other, $\chi_{\mathrm{r}}$ can be recognized to be "polarizability of a bulk matter." When the fundamental relationship ${ }^{12}$ of:

$$
\varepsilon_{0} \varepsilon_{\mathrm{r}} E=\varepsilon_{0} E+P
$$

is introduced, the bulk polarizability is simply related to the relative electric permittivity, $\varepsilon_{\mathrm{r}}$, by:

$$
\varepsilon_{\mathrm{r}}=\chi_{\mathrm{r}}+1,
$$

which simply implies that "a low-permittivity matter has a low bulk polarizability."13 Of note, however, is that the collection of the vectors, $p$, yielding the vector, $P$, depends on the molecular "aggregation structure." In other words, the bulk polarizability cannot simply be related to the molecular polarizability. Even if the molecular polarizability is large, the polarization density can be negligibly small when the induced small dipole-moments are oriented in a deconstructive manner.

A similar logic holds when the Clausius-Mossotti relation (Eq. (9)) is taken into account.

$$
\frac{\varepsilon_{\mathrm{r}}-1}{\varepsilon_{\mathrm{r}}+2} \frac{M}{d}=\frac{N_{\mathrm{A}} \alpha}{3 \varepsilon_{0}}
$$

Here, $M, d$ and $N_{\mathrm{A}}$ are the molecular mass, density, and Avogadro number, respectively. The relation directly attributes the electric permittivity to the molecular polarizability. Regardless, the relation is deduced under an orientation assumption that the collection of $p$ becomes very small ${ }^{12}$ ( $P \approx 0$; Lorentz's local field approximation). The apparent "molecular" polarizability in the equation thus cannot be related to the "bulk" property without considering the aggregation structure. 
According to the SDA model, ${ }^{4}$ in the case of an $\mathrm{Rf}$ compound having a long $\mathrm{Rf}$ group, the

dipole arrays are generated as a result of the strong interactions among the "twisted Rf groups" via the permanent dipoles. Since the dipole "arrays" are oriented with different directions at each $\mathrm{CF}_{2}$ level, a negligibly small polarization density is yielded on a macroscopic average ${ }^{4}$ (Figure 1). The permittivity should thus be small via Eq. (7), which induces a small "bulk polarizability" because of Eq. (8). In short, the Rf groups are aggregated tightly thanks to the permanent dipoles with the twisted orientation resulting in the fluorophilic effect, but the apparent dipole becomes small "over the aggregate" $(P \approx 0)$. This situation corresponds to Lorentz's local field approximation, which readily bridges the single polarizability to the bulk one (Figure 1).

\section{Concluding remarks:}

A perspective to comparatively understand the intrinsic difference between the normal alkyl and $\mathrm{Rf}$ groups is provided with respect to the molecular interactive forces and electrodynamics. The dipole-dipole interaction due to the permanent dipoles plays the primarily important role of making the molecular aggregation structure, which elucidates the high melting temperature, the fluorophilic effect and the low surface energy. The Rf-specific packing, on the other hand, makes the bulk polarizability very small, which enables us to discuss the electric permittivity directly via the molecular polarizability. In this manner, the molecular orientation and packing are found to be key factors to unify the conventional theories. 


\section{Acknowledgment:}

This work was financially supported by a Grant-in-Aid for challenging Exploratory Research

(No. 26620118 (TH)) from Japan Society for the Promotion of Science, from Japan Society for the Promotion of Science.

\section{References:}

1. M.P. Krafft, J.G. Riess, Chem. Rev. 109 (2009) 1714.

2. H.W. Starkweather, Jr., Macromolecules 19 (1986) 1131.

3. K. Skrabania, H. von Berlepsch, , C. Böttcher, A. Laschewsky, Macromol. 43 (2010) 271.

4. T. Hasegawa, T. Shimoaka, N. Shioya, K. Morita, M. Sonoyama, T. Takagi, T. Kanamori, ChemPlusChem 79 (2014) 1421.

5. F. London, Trans. Faraday Soc. 33 (1937) 8.

6. W.H. Keesom, Comm. Phys. Lab. Leiden Suppl., 24B (1912) 23.

7. V.I. Minkin, O.A. Osipov, Y.A. Zhdanov, Dipole moments in organic chemistry (Plenum Press, New York, 1970).

8. K.J. Miller, J. Am. Chem. Soc. 112 (1990) 8533.

9. Calculated and experimental adiabatic ionization energies disclosed by NIST: http://cccbdb.nist.gov/ie1.asp 
10. J.E. Huheey, Inorganic chemistry (Harper\&Row, New York, 1978).

11. R.R. Thomas, Material properties of fluoropolymers and perfluoroalkyl-based polymers.

(Chapter 4) Fluoropolymers 2 (Springer, Heidelberg, 1999).

12. J.D. Jackson, Classical electrodynamics 3rd ed. (Wiley, Hoboken, 1999).

13. W. Groh, A. Zimmermann, Macromol. 24 (1991) 6660.

Acknowledgment: This work was financially supported by Grant-in-Aid for challenging Exploratory Research (No. 26620118) from Japan Society for the Promotion of Science.

Author Information: The authors declare no competing financial interests. Readers are welcome to comment on the online version of the paper. Correspondence and requests for materials should be addressed to T.H. (htakeshi@scl.kyoto-u.ac.jp). 
Table 1 Relative intensities of the orientation, induction and dispersion effects contributing to the van der Waals force between the $\mathrm{C}-\mathrm{H}$ and $\mathrm{C}-\mathrm{F}$ groups. (The unnecessary factor, $-R^{-6}$, is removed from the calculation after London.)

\begin{tabular}{c|ccc|ccc}
\hline & $\mu / \mathrm{D}^{[7]}$ & $\alpha / \mathrm{m}^{3[8]}$ & $\mathrm{IE} / \mathrm{eV}[9]$ & $\begin{array}{l}\text { Orientation } \\
\mathrm{effect} / \mathrm{J} \mathrm{m}^{3}\end{array}$ & $\begin{array}{l}\text { Induction } \\
\mathrm{effect} / \mathrm{J} \mathrm{m}^{3}\end{array}$ & $\begin{array}{c}\text { Dispersion } \\
\mathrm{effect} / \mathrm{J} \mathrm{m}^{3}\end{array}$ \\
\hline $\mathrm{C}-\mathrm{H}$ & 0.40 & $0.652 \times 10^{-30}$ & 10.64 & $\begin{array}{c}0.422 \times 10^{-79} \\
0.209 \times 10^{-79}\end{array}$ & $5.43 \times 10^{-79}$ \\
$\mathrm{C}-\mathrm{F}$ & 1.39 & $0.555 \times 10^{-30}$ & 9.11 & $(6.96 \%)$ & $(3.44 \%)$ & $(\mathbf{8 9 . 6 \% )})$ \\
\hline
\end{tabular}




$$
\frac{\varepsilon_{\mathrm{r}}-1}{\varepsilon_{\mathrm{r}}+2} \frac{M}{d}=\frac{N_{\mathrm{A}} \alpha}{3 \varepsilon_{0}} \quad \begin{aligned}
& \text { SDA helps 'single' be } \\
& \text { connected to 'bulk' }
\end{aligned}
$$

$$
\begin{aligned}
& \text { - low } \varepsilon_{\mathrm{r}} \\
& \text { - low surface energy }
\end{aligned}
$$

Figure 1 T. Hasegawa 